\title{
THE MECHANISM OF THE PAIN IN TRIGEMINAL NEURALGIA
}

\author{
BY \\ ERIC KUGELBERG and ULF LINDBLOM
}

From the Department of Neurology of the Karolinska Institute, Serafimerlasarettet, Stockholm, Sweden

Trigeminal neuralgia (tic douloureux) is characterized by brief paroxysms of pain limited to the facial distribution of the trigeminal nerve and is precipitated by stimuli to sensory endings in the trigeminal receptive area. The condition is relieved by blocking the afferent input of the trigeminal nerve. According to Wyburn-Mason (1953) and Crue, Shelden, Pudentz, and Freshwater (1956), attacks of trigeminal neuralgia can in some cases be abolished by interruption of adjacent cervical nerves.

The neurophysiological mechanism underlying trigeminal neuralgia is still obscure (cf. List and Williams, 1957). King and Meagher (1955), King and Barnett (1957), and King, Meagher, and Barnett (1956) described alterations of trigeminal nerve potentials associated with over-reaction to tactile facial stimulation in cats produced by applying alumina gel or strychnine to the spinal trigeminal nucleus. The electrical potential changes in the human trigeminal system associated with the paroxysmal pain, however, remain to be investigated. Moreover, the finding of a correlation between the clinical syndrome of tic douloureux and the electrophysiological phenomena presupposes a detailed knowledge of the clinical events. Since the mechanism of the paroxysmal pain is intimately associated with afferent discharges we have studied with semiquantitative methods the relation between the attack of pain and stimuli applied to the skin trigger zone. The effect of anti-epileptic drugs, such as hydantoin and lidocain, has also been investigated.

\section{Material and Methods}

The present investigation was carried out on 50 patients with trigeminal neuralgia who were admitted to the Neurological Clinic during the years 1946 to 1957 and in whom the attacks could be evoked by mechanical stimulation of the trigeminal receptive area. A necessary requirement was that the patient had an observant mind and was willing to undergo repeated provocations of the attacks.

One patient, an 18-year-old girl, suffered from "symptomatic" trigeminal neuralgia associated with platybasia. In another patient the neuralgia was associated with an intracranial arterial aneurysm. The remainder had the common " cryptogenic" type.

At the beginning of the investigation the attacks were provoked by stroking the trigger zone with the finger tip at, so far as possible, a constant rate, speed, and pressure. Later, a vibratory stimulator was employed, consisting of a pin attached to the membrane of a loud-speaker which led to a Grass stimulator. The head of the pin, which was a round knob, was pressed against the skin. The frequency and amplitude of the vibrations were controlled with the stimulator which was switched on and off manually. The amplitude ranged from 0 to $1 \mathrm{~mm}$. and reached its maximum within $2 \mathrm{~m} . \mathrm{sec}$. This provided a comparatively well-defined and reproducible mechanicas stimulus of the skin.

\section{Results}

Some patients have, during particularly severe periods of the disease, attacks of pain which apparently start spontaneously. They occur withouk tangible external irritation, such as touching the face, talking, or eating, which is usually necessary for precipitating an attack during less active periods. The same patients may have periods of remission when not even external stimuli will provoke an attack. It is also a well-known fact that the severity of the disease can vary from day to day or from hour to hour.

Since even in patients with "spontaneous" attacks the pain is relieved by blocking the afferent pathway of the fifth nerve, it may be assumed that the pain is precipitated by afferent discharges and that deterioration or improvement reflects a lowering or raising of the attack threshold. During periods of "spontaneous" attacks a minimum stream of afferent impulses in the trigeminal nerve is evidently sufficient to provoke an attack. During periods of improvement a higher intensity of afferent impulses is required, and during periods of remission the threshold is not reached even with a high intensity of afferent discharge.

The rapid fluctuation in the severity of the symptoms indicates that the neurophysiological state responsible for the tic syndrome is very unstable. Accordingly the quantitative relationship 


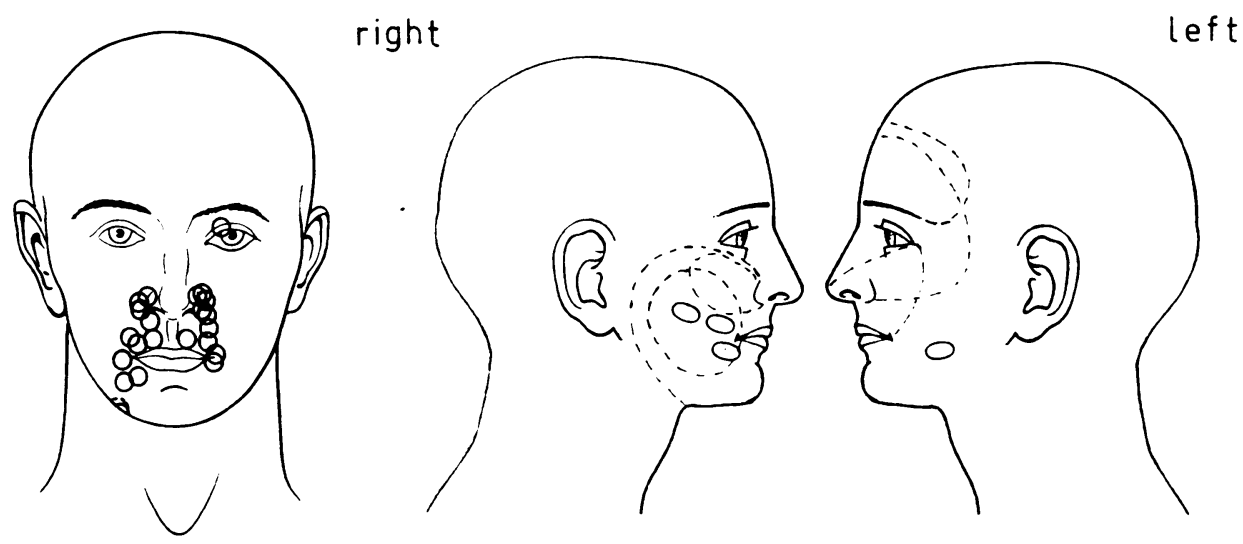

FIG. 1.-Distribution of 31 trigger zones in 30 patients. Circles denote circumscribed and broken lines widespread, cutaneous trigger zones. Ovals indicate localization to alveolar gingiva.

between the paroxysmal pain and the afferent discharge may be expected to vary not only in different individuals but also in the same individual at different examinations as well as during the course of a prolonged examination. A basic pattern of the trigger mechanism, however, may be distinguished.

The Trigger Zone.-The site of the trigger zone in the last 30 patients examined is shown in Fig. 1. Although the trigger zone could be located almost anywhere within the trigeminal receptive area it was most common in the nasolabial fold, on the upper lip, the lateral part of the lower lip, and the alveolar gingiva. Some patients had two trigger zones and some reported that their trigger zone had shifted from one point to another during the course of the disease. One patient stated that she had a trigger zone outside the trigeminal area, located in the ipsilateral axilla. At the time of examination, however, it was not present, and therefore the information is uncertain. Crue et al. (1956) reported a case of trigeminal neuralgia with a trigger zone on the big toe.

In some of the patients the trigger zone was large and diffuse but in the majority it was comparatively small and well-defined. How small the trigger zone can actually be is difficult to determine, but in some of our patients it was not larger than 1 or $2 \mathrm{sq}$. $\mathrm{mm}$. There was no obvious relationship between the severity of the attack and the size of the trigger zone. Very severe attacks could be fired in patients with an almost punctate trigger zone as well as in those with a large trigger zone. The pain usually started near the trigger zone. Although it was rather difficult for the patients to determine accurately the exact spot in which the pain actually started, it was obvious that the pain did not always start in the trigger zone. For example, in one patient with the trigger zone on the upper eyelid the pain started in the cheek ( $c f$. List and Williams, 1957).

Adequate Stimulus.-It is common knowledge that the attacks of pain are precipitated by tactile stimulation of the trigger zone (e.g., touching the face, as in shaving or washing) or by stimuli such as talking and mastication which increase the proprioceptive inflow. Pain is a less effective stimulus. In our patients severe pinching of the skin of the trigger zone with forceps failed to evoke an attack. Thermal stimuli (a warm metal rod or cube of ice) were ineffective when applied slowly, as was slowlyapplied firm pressure. On the other hand, sudden application or release (off-effect) of pressure evoked an attack in susceptible individuals. This suggests fairly rapid adaptation of the receptors responsible for the paroxysmal pain. Stroking the skin with a blunt object, e.g., the finger of the examiner, was a highly effective stimulus. Firm stroking is perceived as touch, light stroking as touch and tickle. Rapid displacement of a few hairs may also be effective. This is perceived as a tickle, with or without the component of touch. Slow displacement of the hair, however, or even a slow, steady, painful pull on the hair failed to fire an attack. The vibratory stimulus, causing a rapid distortion of the skin, was effective in susceptible individuals.

Electrical stimulation was employed in an effort to determine which fibres convey the effective impulses. In two patients with the trigger zone on the upper lip a needle electrode was inserted into the infraorbital foramen. Repetitive weak shock stimuli to the maxillary nerve gave rise to the vibratory sensation typical of tactile fibre stimulation ( $c f$. Kugelberg, 1944), but did not evoke a paroxysm of pain. An increase of the stimulus strength to 
threshold for the larger pain fibres still failed to provoke an attack. These results indicate either that the fibres responsible for the paroxysmal pain have a smaller diameter and a higher threshold than the large pain fibres or possibly that the spatial and temporal pattern of the triggering discharge is too specific to be imitated by electrical shock stimulation of the nerve. Shock stimulation did not appear to have an inhibitory action since the attack could still be triggered from the upper lip during the electrical stimulation.

Eliciting the Attack of Pain.-Four factors are discussed.

(1) Minimal Afferent Discharge.-In three patients it was possible to trigger an attack by rapid displacement of a single hair. In susceptible individuals evidently only a minimum number of nerve fibres are required to convey impulses to the critical structure to fire an attack. This does not, however, imply that these were the only fibres responsible for the attack. A proper background discharge is probably also necessary.

(2) Spatial and Temporal Summation.-Provided the trigger zone was not too small, it was possible to show that spatial summation of impulses from a larger area triggers the paroxysm of pain at a lower stimulus strength and at a shorter latency than if a small area is stimulated. In susceptible individuals however, a larger spatial summation does not seem to be a prerequisite for evoking an attack. As mentioned earlier, displacement of a single hair was sometimes sufficient to evoke an attack.

A temporal summation of impulses was evident in all but a few of the patients. In one of these displacement of a few hairs of the nostril by a single pulse of the vibrator evoked a stab of pain, which was accompanied by a twitch of the face with a latency of $57 \mathrm{~m} . \mathrm{sec}$. (Fig. 2). In a few patients a single touch of the trigger zone similarly elicited a stab of pain with a very short latency. Since the patients did not react with a well-defined twitch

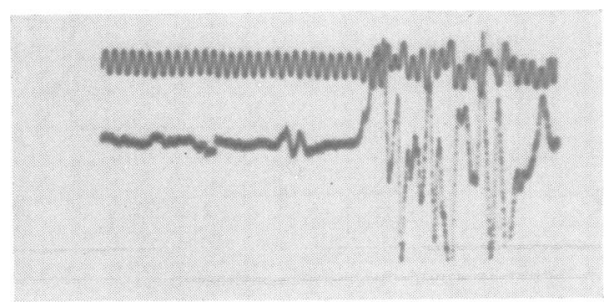

Fig. 2.-Electromyogram from the orbicularis oris. Latency of facial twitch accompanying a stab of pain evoked by a sudden displacement of hairs of the nostril. The stimulus started the cathode ray sweep. Time: $500 \mathrm{c} . / \mathrm{sec}$.

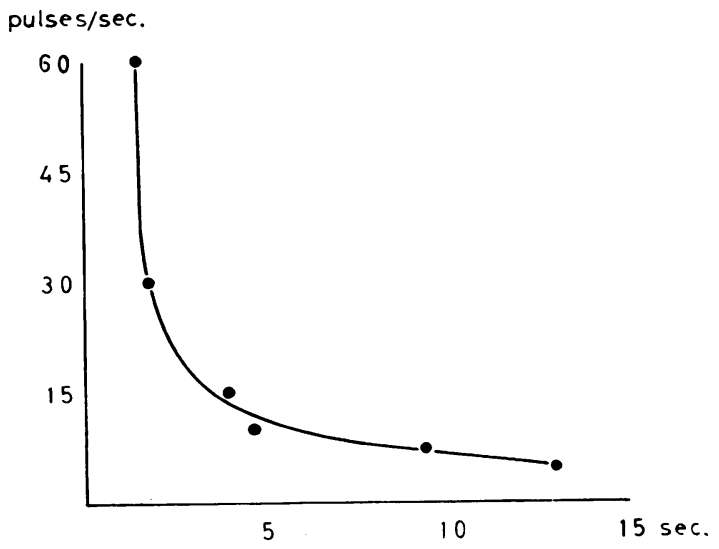

FIG. 3.-Summation time (abscissa) in relation to vibration frequency (ordinate).

of the face it was not possible to measure the latency time accurately.

In the majority of the patients, however, it was necessary to continue the stimulation for a longer period in order to elicit pain. The length of the period was largely dependent on the stimulus intensity. A typical example is shown in Fig. 3 Vibratory stimuli at the rate of 7 per second evoke an attack after a summation time of $15 \mathrm{sec}$. Bg increasing the frequency to 30 vibrations per secon $\bar{Q}$ \& the summation time was gradually reduced to aboug 1.5 sec. Further increase in frequency did noe further reduce the latency time. Variations solel $\overrightarrow{5}$. in the amplitude of the stimuli had the same effect of on the latency. A summation time up to $30 \mathrm{sec}$. was observed with weak stimulation.

The results indicate that the excitatory state necessary to fire an attack may be built up over a considerable time by temporal summation of afferent impulses.

One may ask how quickly the effect of the stimulus subsides. To test this the trigger threshold was determined at different intervals after a conditioning stimulus. The minimum duration of a stimulus which fires a brief jab of pain was estimated. A test stimulus of equal intensity was delivered $5,10,15$, or 25 seconds after the conditioning stimulus and the duration necessary to reach the trigger threshold was measured. The quotient of the duration of the test stimulus and the conditioning stimulus is plotted against the stimulus interval in Fig. 4. Each point in the figure is the mean of three or four pairs of measurements.

The raised excitability evidently falls rather sharply after cessation of the stimulus but may o remain above the resting value for 20 to $25 \mathrm{sec}$. The $\mathrm{N}$ raised excitability in this experiment was not due N 


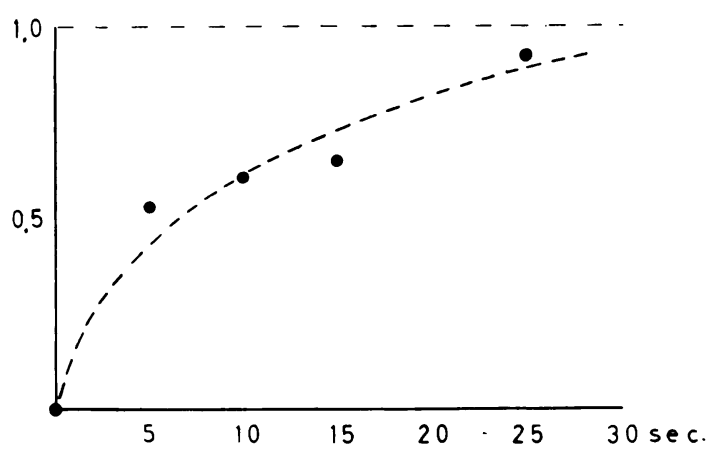

FIG. 4.-Decay of excitability following a conditioning skin stimulus to the trigger area. The quotient of the duration of the test stimulus and the conditioning stimulus (ordinate) is plotted against the stimulus interval (abscissa).

to the jab of pain since the same result was obtained if the conditioning stimulus was just subthreshold for triggering the pain. Experiments of this type are difficult and can be performed only in subjects with a stable trigger threshold. The result was consistent in three out of five patients tested.

(3) Effect of Stimulation.-Stimulation of the trigger area in our patients resulted in: (a) Paraesthesia in the form of pins and needles or tingling (this was uncommon); (b) a jab of pain which may recur in rapid succession when the stimulation is continued; $(c)$ sustained pain which continues when the stimulus is prolonged but does not outlast the stimulus; $(d)$ sustained pain which continues even after the stimulus is discontinued.

More than one type may occur in one and the same individual although one type usually predominates. During periods of improvement the response in $(d)$ may often be supplanted by the $(c)$ type.
The pain reaches its maximal intensity rapidly, usually within a few seconds, remains stable for a variable period, and then gradually subsides. Alterations in the intensity are accompanied by variations in the spatial distribution of the pain. The pain spreads rapidly at the baginning of the attack but recedes more slowly at the end of the attack, generally in the reversed order of its evolution. Some patients complain of tenderness to pressure in the painful area during an attack that is not severe enough to mask the tenderness. In our patients the attacks seldom lasted longer than two to three minutes.

(4). Refractory Period.-All clinicians who have treated a large number of patients with trigeminal neuralgia will have encountered those who relate that immediately after an attack they can safely go on eating and talking for a while without running the risk of a new attack. This, of course, indicates that a refractory period follows the attack. The time relations, however, do not appear to have been investigated.

The patient, E. J., had, before trigeminal rhizotomy, during the course of one day's observation, spontaneous attacks of severe pain of 30 to $60 \mathrm{sec}$. duration at fairly regular intervals, with free intervals of one and a half to two and a half minutes' duration (Fig. 5, upper diagram). Since the paroxysms presumably were evoked by a more or less continuous stream of minimal afferent discharges in the trigeminal nerve, the intermittent response to continuous stimulation is presumptive evidence of a refractory phase. To test this, the intensity of the afferent discharge was increased by a series of light strokes applied with the finger-tip to the trigger zone on the patient's cheek at the rate of three strokes per second. This reduced the interval between the attacks to about 50 seconds (Fig. 5, middle diagram). If, in
FIG. 5.-Duration of the free interval between attacks. Upper diagram, in a series of spontaneous attacks; middle diagram, attacks provoked by continuous stroking of the trigger area at the rate of 3 per sec.; lower diagram, stroking at the rate of 6 per second.
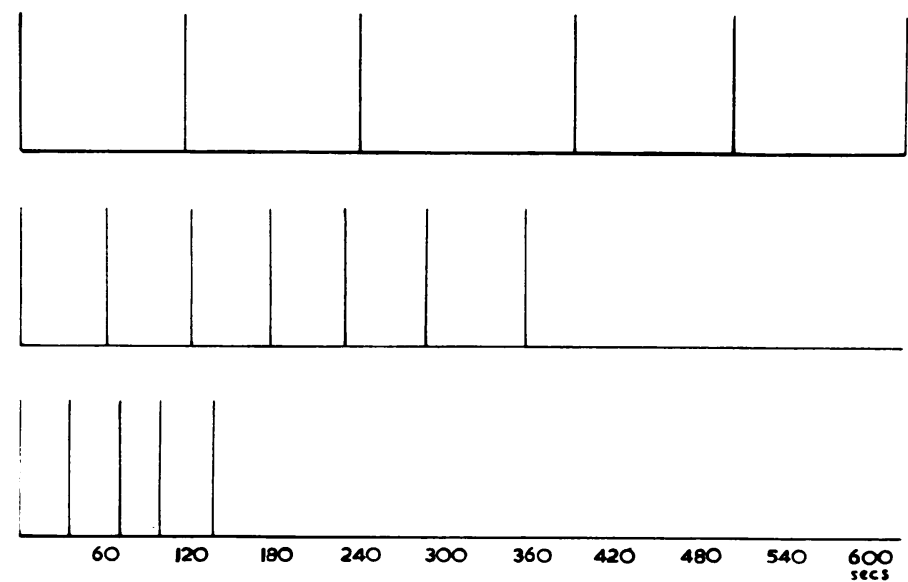
FIG. 6.-Refractory phase of a pain paroxysm tested by stroking the trigger area intermittently for periods of 10 sec. Filled in area denotes attacks, vertical lines a stab of pain. The interrupted line below the base line represents 10-second periods of stimulation.

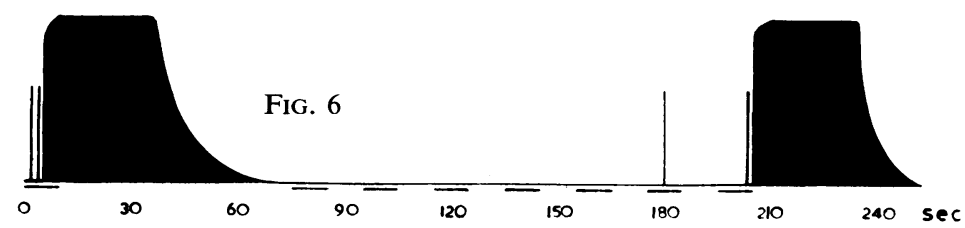

order further to increase the afferent discharge, the strokes were applied at the rate of six per second, the free intervals were reduced to about 30 seconds (Fig. 5, lower diagram). Further increase in the intensity of the afferent discharge by strokes applied at a maximal speed and frequency reduced the free interval but little. It could not be reduced to less than 20 seconds, and the attacks continued to come rhythmically during all forms of stimulation.

If the threshold for eliciting an attack which follows an antecedent attack is tested by a more intermittent stimulation, a similar condition is found, as exemplified in the patient $H$. S. This

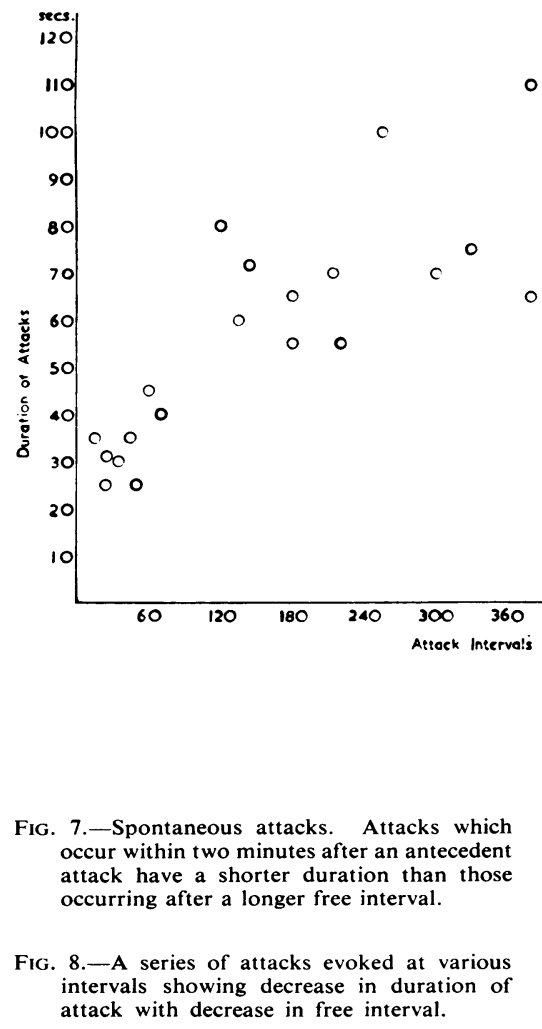

patient had no spontaneous paroxysms but light stroking of the chin at the rate of three strokes per second for a period of 10 seconds regularly elicited a very severe attack of pain of about two minutes' duration. If the stroking was repeated at 20 -second intervals after an antecedent attack, the next attack was evoked first after about two minutes (Fig. 6). To reduce the interval between the attacks required intensification of the stimulus. Here also it was not possible to reduce the free interval to less than 20 seconds. The result was the same if only one 10 -second period of stimulation was interpolated after each antecedent attack but at varied intervals.

From these examples it is clear that the attacks of pain are followed by a refractory phase during which the threshold is raised. At first the refractory phase is absolute for the stimuli tested and excitability returne gradually to its former level two tQ three minutes after the end of a severe attack.

Furthermore, the attack followin within the refractory phase of ape antecedent attack tends to be dimin? ished in duration and intensity and shows, possibly, less tendency to spread. This is exemplified in the patient E. K., who periodically had severe attacks at irregular intervals. Fig. 7 shows the duration of the individual "spontaneous" attacks

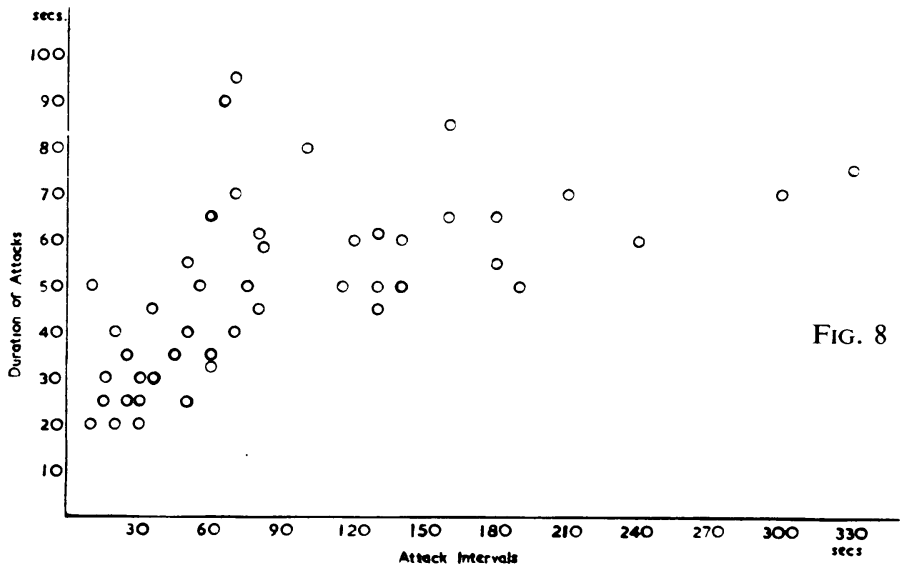


plotted against the duration of the free interval which preceded the attack. If the free interval was less than two to three minutes, the paroxysm decreased in duration with decrease in length of free interval. This was also the case if, when the patient had no spontaneous attacks, a test attack was interpolated at varied intervals after a similarly provoked antecedent attack (Fig. 8). Moreover, it was again observed that no spontaneous paroxysms occurred at intervals of less than 20 seconds, nor was it possible to evoke them earlier (Fig. 6-8).

In paroxysms occurring within the refractory phase after an antecedent attack, diminution in the intensity of the pain is not so obvious as the decrease in the duration of the paroxysm. Some patients admit a considerable decrease in the intensity of the pain while others do not. It is possible that the discharge of pain impulses during the paroxysm is so intense that discrimination is lost.

It is desirable at this point to establish that the refractory phase is dependent chiefly on the attack of pain and not on the stimulus that precipitates it. With the rough methods of evaluation used here no refractory phase could be detected after a series of stimuli to the skin for periods of 10 seconds to two minutes at an intensity just below threshold. Nor did a stimulus of 60 to 70 seconds' duration applied at the beginning of the refractory period alter the rhythm of the attacks occurring spontaneously at regular intervals. The intensity of the stimulus used was sufficiently strong to provoke an attack at the end of the refractory period.

The duration of the refractory phase is a function of the duration as well as of the intensity of the preceding attack. Attacks of a few seconds' duration show hardly any refractoriness as tested by the vibrator. Furthermore, in two patients who had paroxysms of only slight pain, it was possible, by stimulation of the trigger area, to maintain an attack for 19 minutes during which time no absolute refractory period occurred. The pain, however, steadily decreased in intensity during the attack and was finally replaced by a sensation of glowing warmth. Conversely, the duration of the refractory phase could be increased by provoking a series of attacks in rapid succession. Although in this case the duration of the attacks decreases and consequently the duration of the refractory period, the sum effect of the series may be an increase.

In the patient E. K., who at one time had fairly regular spontaneous attacks, each series of provoked attacks was followed by a compensatory pause.

Effect of Anti-epileptic Drugs.-Of the different drugs used therapeutically, novocaine has been reported to be effective not only when given as a

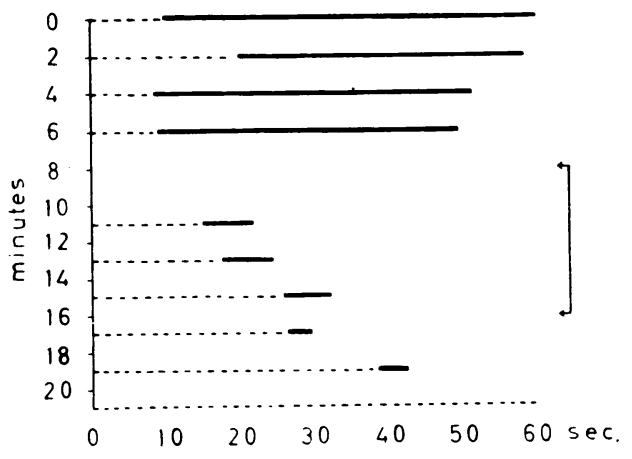

FIG. 9.--Increase in summation time (broken line) and decrease in duration of pain (thick solid line) following intravenous injection of lidocaine (between arrows), $2 \mathrm{mg} . / \mathrm{kg}$. Vibratory stimulation at two-minute intervals before (time 0-6) and after (time 11-21) the beginning of the injection.

local anaesthetic for blocking conduction in the peripheral trigeminal pathway, but also when injected into the systemic circulation in comparatively small doses (see Kubànyi, 1956).

In the present investigation lidocaine was given intravenously to six patients and its effect tested by vibratory stimulation of the trigger area at regular intervals which were longer than the refractory period. In all six patients lidocaine, in a dose of 2 to $4 \mathrm{mg}$. $/ \mathrm{kg}$. body weight, resulted in partial or complete relief of the pain paroxysms. Fig. 9 illustrates a typical result obtained in the patient N. L., in whom attacks could regularly be evoked before the injection. It is seen that during the course of the injection the summation time increases and the duration of the pain paroxysm decreases until complete abolition is obtained. Owing to the rapid elimination of the drug (Sung and Truant, 1954), however, the effect is only temporary and the attacks usually regained their original intensity one hour after the injection.

Several workers have found that diphenylhydantoin given orally is of benefit in the treatment of trigeminal neuralgia (see Kubànyi, 1956; Berczeller and Szecsödy, 1956). In the present series five patients were given intravenous diphenylhydantoin (" epanutin ": Parke Davis) in a dose of 3 to $5 \mathrm{mg}$./ $/ \mathrm{kg}$. The results were about the same as with lidocaine, although the number of patients is too small to permit a detailed comparison. The total relief of pain obtained with intravenous diphenylhydantoin was short-lived, but the patients stated that the attacks were less intense and less frequent during the rest of the day. Four of these patients were also given barbiturates. A solution of diemal and allypropymal ("hypnofen": ACO) was given 
intravenously until the patient became drowsy, i.e., a dose of 5 to $10 \mathrm{mg} . / \mathrm{kg}$. As a rule attacks of maximum intensity and duration could still be elicited after the injection.

Thus the results obtained in acute experiments and controlled by measurements of the trigger threshold confirm the clinical observations reported earlier.

\section{Discussion}

The adequate stimulus for eliciting an attack of pain seems to be distortion of the hair, skin, or mucosa. Our observations indicate a fairly rapid adaptation of the critical receptors engaged by such stimuli. Since pain and thermal stimuli were largely ineffective it may be concluded that the afferent discharge which triggers the pain arises from receptors responding to touch.

However, it must be borne in mind that touch stimuli give rise not only to a sensation of touch but also to a sensation of tickle. In fact, the threshold for tickle is lower than that for touch and even if a firm touch is not perceived as tickle it is clear that afferent discharges from receptors responsible for tickle must be evoked. There is no general agreement on the relationship between the tickle sensation and the touch or pain sensation (Graham, Goodell, and Wolff, 1951). These authors consider that tickle is mediated by the same nerve fibres as itch and pain. It seems to be established that the tickling sensation in the trigeminal area is subserved by fibres entering the spinal $\mathbf{V}$ tract and nucleus, since it is abolished by trigeminal tractotomy which leaves the components of touch more or less intact (Zotterman, 1939). Our experiments do not exclude the possibility that the receptors responsible for tickle play an important role in initiating the pain paroxysm.

The negative results of electrical stimulation suggest that the effective impulses are not transmitted by the largest touch fibres.

As shown by Zotterman (1939) in his fundamental studies on the cat, a light touch evokes action potentials in delta fibres, usually followed by action potentials of still slower fibres, probably belonging to the $\mathrm{C}$ group, i.e., fibres earlier considered to subserve pain. A firm touch engages fibres from the largest down to the smallest. Douglas and Ritchie (1957) have confirmed with another technique the findings of Zotterman. Indeed these authors consider that the bulk of delta and unmyelinated fibres mediate touch rather than temperature and pain. Thus it may be concluded that the fibres engaged by touch stimuli have a broad calibre spectrum. It is believed that the largest fibres of the trigeminal nerve chiefly go to the main sensory nucleus and the delta (cf. Sjöqvist, 1938) and $z$ unmyelinated fibres to the spinal tract nucleus. Thus a skin stimulus that evokes pain paroxysms feeds impulses not only into the main nucleus but to a large extent also to the spinal nucleus.

The summation time and the duration of the attacks varied considerably in our series. Whether there is a qualitative or merely a quantitative difference in the mechanisms underlying the short latency jab of pain and the attacks that requires a long summation time is not clear.

The short latency jab of pain may possibly be explained by a simple interaction or short circuiting of, for example, the touch and pain fibres. Animal experiments have shown that such an artificial synapse has a delay that is exceedingly short (Arvanitaki, 1942; Granit and Skoglund, 1945; Skoglund, 1945). In one of our patients, the motor response to skin stimulation which evoked a pain paroxysm had a latency of about $60 \mathrm{~m} . \mathrm{sec}$. The bilateral distortion of the face resembled a startle response which in fact has a similar latency. Thus, if the twitch were the result of the pain, the latter must have very short latency.

In most cases, however, a more or less longe lasting attack was fired after a considerably longe summation time. A summation time of 15 to 30 seconds was not uncommon. It is highly probable that the summation takes place in the structure responsible for the attack rather than ing the receptors. The characteristic refractory perios of up to two to three minutes after an attack is $\overrightarrow{0} \overrightarrow{0}$ scarcely the result of a refractory phase in the $f$ receptors since it was shown to be a function of the intensity and duration of the attack and independent of skin stimuli strong enough to discharge the receptors. It would seem that an excitatory state is gradually built up by a temporal and spatial summation of afferent impulses and when it reaches a critical level the attack is fired. This process would be expected to be accompanied by a slow potential change. A slow change does not fit in with the properties of an artificial synapse between nerve fibres nor has a similar phenomenon been reported from pathological ganglia in animal experiments, although the latter has admittedly been little investigated. It is, however, of the type encountered in central structures.

Once an attack is fired it frequently outlasts the stimulus which initiated it. The stimulus itself does not have such a long-lasting effect, since it was shown that the excitability, as reflected by the trigger threshold, diminishes fairly rapidly after the stimulus is withdrawn. The cause of the prolonged maintained discharge would therefore be an event that is started by the attack. This event is a strong 
excitant since it may keep a very strong pain alive for one to two minutes and when it has lost its potency skin stimuli, no matter how strong, often cannot revive the attack.

The refractory phase is certainly intimately associated with the paroxysmal character of the pain and responsible for the cutting short of the attack. Here again slow changes are encountered in the excitability of the mechanism underlying the pain.

The pathologically altered structure that is responsible for the trigeminal neuralgic syndrome is most likely located in the brain-stem, as also was thought by Crue et al. (1956) and List and Williams (1957). A thalamic origin of trigeminal neuralgia as proposed by Lewy and Grant (1938) seems unlikely since it is improbable that a lesion at this level could account for such a spatially limited pain phenomenon. The interesting observations of King et al. (1955, 1956, 1957) in animal experiments are at present difficult to apply to the phenomena we have studied since the potential changes in the cat proceed on a time scale in milliseconds as compared to seconds and minutes in man.

The fact that anti-epileptic drugs, such as hydantoin and lidocaine (cf. Bernhard and Bohon, 1955; Bernhard, Bohon, and Höjeberg, 1955), raise the trigger threshold for the pain favours the theory of a central origin. In fact Trousseau has already designated tic douloureux " neuralgia epileptiforme". It does not, however, prove a central origin of the pain since both drugs also act on peripheral nerves (Bernhard and Valleala, 1958; Morrell, Bradley, and Ptashne, 1958).

If it is correct that the origin of the pain in most cases of trigeminal neuralgia is located centrally the favourable effect of Taarnhøj's (1952) operation seems to be explained by a blocking of critical fibres and a resultant reduction of the afferent discharge essential for eliciting the pain paroxysm.

\section{Summary}

An investigation has been made in 50 patients with trigeminal neuralgia of the relationship between stimuli applied to the trigger zone and the pain paroxysm. The results are as follows:-

The adequate stimulus for precipitating an attack is touch and possibly tickle, rather than pain or temperature. There is evidence that the largest touch fibres are not involved. This is discussed in relation to the fact that the fibres engaged by touch stimulus have a broad calibre spectrum and therefore feed impulses not only into the main trigeminal nucleus but also to a large extent into the spinal $\mathrm{V}$ nucleus.

Rapid displacement of a single hair was sometimes sufficient to provoke an attack but in most cases a larger spatial summation of afferent impulses was necessary.

A temporal summation of impulses over a considerable time was in the majority of cases necessary to trigger an attack. Summation times of 15 to 30 seconds were often observed, provided the stimulus was weak. In some cases, however, the summation time was short.

Cessation of the stimulus is followed by a rapid fall in excitability, although it may remain above resting value for up to 20 seconds after the stimulus, as seen in three patients.

The attack is followed by a refractory period of up to two to three minutes, depending upon the duration and intensity of the pain. Attacks which occur within the refractory phase of an antecedent attack tend to be shorter.

Anti-epileptic drugs, such as lidocaine and hydantoin, were found to raise the attack threshold as well as to shorten the duration of the attack by diminishing its tendency to self-maintenance.

The long summation times, the tendency of the attack to be self-maintained, the effect of antiepileptic drugs as well as the long-lasting refractory period, suggest that the mechanism responsible for the paroxysmal pain is situated centrally, probably in the brain-stem in structures related to the spinal $\mathrm{V}$ nucleus.

\section{REFERENCES}

Arvanitaki, A. (1942). J. Neurophysiol., 5, 89

Berczeller, E., and Szecsödy, E. (1956). Nervenarzt, 27, 369

Bernhard, C. G., and Valleala, P. (1958). Exp. Cell. Res., Suppl. 5. 201

and Bohon, E. (1955). Brit. J. Pharmacol., 10, 288.

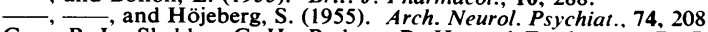

Crue, B. L., Shelden, C. H., Pudenz, R. H., and Freshwater, D. B. (1956). Neurology, 6, 196.

Douglas, W. W., and Ritchie, J. M. (1957). J. Physiol. (Lond.). $139,385$.

Graham, D. T., Goodell, H., and Wolff, H. G. (1951). J. clin. Invest. 30, 37.

Granit, R., and Skoglund, C. R. (1945). J. Physiol. (Lond.), 103, 435.

King, R. B., and Barnett, J. C. (1957). J. Neurosurg., 14, 617.

- , and Meagher, J. N. (1955). Ibid., 12, 393.

Kubànyi, E. (1956). Trigeminusneuralgie. Ürban \& Schwarzenberg. Vienna.

Kugelberg, E. (1944). Acta physiol. scand., 8, Suppl. XXIV.

Lewy, F. H., and Grant, F. C. (1938). Arch. Nelurol. Psychiat. (Chicago), 40, 1126. List, C. F., and Williams, J. R. (1957). A.M.A. Arch. Neurol. Psychiat..

Morrell, F., Bradley, W., and Ptashne, M. (1958). Neurology. 8, 140.

Sjöqvist, O. (1938). Acta psychiat. (Koh.), Suppl. XVII, 139.

Skoglund, C. R. (1945). J. Neurophysiol., 8, 365 . Sung, C. J., and Truant, A. P. (1954). J. Pharmacol. exp. Ther.,

Taarnhøj, P. (1952). J. Neurosurg., 9, 288.

Wyburn-Mason, R. (1953). Brit. med. J., 2, 119

Zotterman, Y. (1939). J. Physiol. (Lond.), 95, 1. 